\title{
Influence of Thermo-Diffusion and Heat Source on MHD Free Convective Radiating Dissipative Boundary Layer of Chemically Reacting Fluid Flow in a Porous Vertical Surface
}

\author{
Mohammed Ibrahim Shaik ${ }^{1^{*}}$ and Suneetha Karna ${ }^{2}$ \\ ${ }^{1}$ Dept. of Mathematics, Gitam Institute of Technology, GITAM University, Vishakhapatnam, Andhra Pradesh \\ 530045 , India. \\ ${ }^{2}$ Dept. of Mathematics, Priyadarshini College of Engineering \& Technology, Nellore, Andhra Pradesh 524004, \\ India \\ Email: ibrahimsvu@gmail.com
}

\begin{abstract}
The article is focused on the effects of Soret and chemical reaction on MHD free convection flow through a vertical porous surface in the presence of heat source, viscous dissipation and radiation. The dimensionless governing equations for this investigation are solved analytically using perturbation technique. Numerical evaluation of the numerical results is performed and the results for velocity, temperature and concentration profiles within the boundary layer are discussed and shown graphically. Also skin-friction coefficient due to velocity, Nusselt number coefficient due to temperature and Sherwood number coefficient due to concentration fields are evaluated.
\end{abstract}

Keywords: Boundary layer; MHD; Radiation; Viscous dissipation; Chemical reaction; Permeability of porous; Heat source.

\section{$1 \quad$ Introduction}

Owing to their numerous applications in the industrial manufacturing process, the problem of heat and mass transfer in the boundary layers of a continuously moving semi-infinite flat plate has attracted the attention of researchers for the past three decades. Some of the application areas are hot rolling, study production, metal spinning, drawing plastic films, glass blowing, continuous casting of metals and spinning of fibers. Annealing and thinning of copper wire is another example. In all these cases, the quality of the final product depends on the rate of heat and mass transfer at the moving surface. By drawing the strips in an electrically conducting fluid subjected to a magnetic field the rate of cooling can be controlled and the final products of the desired characteristic might be achieved. Sakiadis [1] studied the boundary layer of viscous fluid on the continuous moving solid surface. Gribben [2] investigated the MHD boundary layer flow over a semi-infinite plate with an aligned magnetic field in the presence of pressure gradient. Free convection boundary layers and the study on a uniformly heated vertical plate was presented by Goldstein and Eckert [3]. Beard and Walters [4] proposed the two-dimensional boundary layer flow for an elastico-viscous fluid near a stagnation point.

In the present years, the free convective flow of heat and mass transfer problems in the presence of magnetic field through a porous medium have attracted the attention of a number of research scholars because of their possible application in many branches of science and technology such as fibre and granular insulation, a geothermal system, etc. In engineering sciences, it finds many applications in MHD pumps, MHD bearing, MHD power generators, etc. The effects of free convection on stokes problem for a vertical plate under the action of transversely applied magnetic field with heat and mass transfer was discussed by Soundalgekar et al.[5]. Thermal diffusion and natural convection between inclined plates in a porous medium with MHD was analyzed by Raju et al. [6]. Ahmed [7] discovered the radiating fluid and an induced magnetic field over a porous vertical plate. Samira et al. [8] derived free convection MHD flow of an elastoviscous fluid past an infinite vertical plate. Chowdhury and Islam [9] presented the effects of heat and mass transfer on MHD free convective flow of visco-elastic fluid past an infinite vertical porous plate. 
Radiative heat and mass transfer play an important role in manufacturing industries for the design of fins, steel rolling, nuclear power plants, gas turbines and various propulsion devices for aircraft, missiles, satellites and space vehicles are examples of such engineering applications. The effect of radiation on MHD free convective heat and mass transfer flow becomes more industrially important. Radiation effect on the natural convective flow of a gas past a semi-infinite plate was studied by Soundalgekhar and Takhar [10]. Raju et al. [11] investigated the combined effect of heat and mass transfer on a free convective flow through a porous medium bounded by a vertical surface with radiation. Radiation effect on free convective flow over a porous vertical plate was studied by Hossain et al.[12]. Radiation effect on MHD free convective flow on the vertical porous plate using finite difference method was proposed by Abd E Naby et al. [13]. Radiation effect on the three-dimensional flow in a vertical channel subjected to a periodic suction was discovered by Guria et al. [14]. Rajesh and Vijaya Kumar Varma [15] studied heat and mass transfer effects on radiating MHD free convection flow past an exponentially accelerated vertical plate with variable temperature. The heat transfer in a porous medium with high porosity in the presence of radiation was analyzed by Raptis [16].

Convective flows with simultaneous heat and mass transfer under the influence of magnetic field and chemical reaction arises in many natural and artificial transfer process in many branches of science and engineering applications. This phenomenon plays an important role in the chemical industry, power and cooling industry for drying, chemical vapor decomposition on surfaces, cooling nuclear reactor and petroleum industry. The effects of chemical reaction and thermal radiation on steady MHD free convective boundary layer flow through a porous vertical surface with constant suction was investigated by Raju et al. [17]. Satya Narayana and Sravanthi [18] proposed the influence of variable permeability on unsteady MHD convection flow past a semi-infinite inclined plate with thermal radiation and chemical reaction. The effect of the chemical reaction on the process of an unsteady flow past a vertical plate with a constant heat flux was proposed by Das et al. [19]. Satya Narayana [20] analyzed the effects of chemical reaction and thermal radiation on MHD free convection flow past an infinite vertical plate with variable suction and heat source.

The study of heat generation or absorption effects in moving fluids is important in view of several physical problems such as fluids undergoing exothermic or endothermic chemical reaction. Mohamed et al. [21] presented the effects of radiation and chemical reaction on MHD free convective flow of a polar fluid through a porous medium with heat generation. The problem of free convective flow along a vertical wavy surface embedded in a saturated porous media in the presence of internal heat generation or absorption effect was presented by Hady et al. [22]. Alam et al. [23] noticed the heat and mass transfer effects on MHD free convective flow past an inclined semi-infinite heated surface in the presence of heat generation. The heat and mass transfer effects on MHD flow over a moving permeable cylinder with heat generation or absorption and the chemical reaction was discovered by Chamkha [24].

In all the studies mentioned above, viscous dissipation is neglected. But, viscous dissipation in free convective flow is an important factor when the flow field is of extreme size or in the high gravitational field. Viscous dissipation effect on steady free convective heat and mass transfer flow past a semi-infinite flat plate was studied by Geetha and Moorthy [25]. The thermal radiation effect on hydromagnetic free convection flow past an impulsively started vertical plate with variable surface temperature and concentration by taking into account the heat due to viscous dissipation was presented by Suneetha et al. [26]. Satya Narayana [27] reported the effects of thermal radiation and the viscous dissipation on an unsteady MHD convection flow past a semi-infinite vertical permeable moving porous plate. The influence of viscous dissipation effect in natural convective flows, showing that the heat transfer rates are reduced by an increase in the dissipation parameter was noticed by Mahajan et al. [28].

The Soret effect arises when the mass flux contains a term that depends on the temperature gradient. The major focus of our investigation is the effect on free convection of the addition of a second fluid. of a second additional fluid on free convection. Convection in binary fluids is considerably more complicated than that in pure fluids. Both temperature and concentration gradient contribute to the initiation of convection and each may be stabilizing or destabilizing. Even when a concentration gradient is not externally imposed (the thermo solutal problems) it can be created by the applied thermal gradient via the Soret effect. Mohammed Ibrahim and Suneetha [29] proposed the Soret and chemical reaction effects on MHD flow of a visco-elastic fluid through an impulsively started infinite vertical plate in the presence of heat source. Bhavana et al. [30] studied the effect of the Soret on MHD free convective flow over a vertical plate in the presence of heat generation. Soret and chemical reaction 
effects on hydromagnetic free convective Walter's memory flow in the presence of constant suction and heat sink was investigated by Pavan kumar et al.[31]. Ahmed [32] discussed the heat and mass transfer effects on Hartman flow in the presence of constant heat source and Soret. The same author Ahmed [33] explained the effects of Soret and radiation on transient MHD free convection from an impulsively started infinite vertical plate.

However, to the best of the authors' knowledge, no attempt so far has been made to analyze the heat source and Soret effects on a steady MHD flow of viscous fluid through a porous medium bounded by a porous surface subjected to suction with a constant viscosity in the presence of radiation and chemical reaction.

\section{$2 \quad$ Mathematical Model}

We consider a viscous incompressible, electrically conducting and radiating fluid through a porous medium occupying a semi-infinite region of the space bounded by a vertical infinite surface. The $x^{*}$ axis is taken along the surface in an upward direction and the $y^{*}$ axis is normal to it. A uniform magnetic field $B_{0}$ is assumed to be applied in a direction perpendicular to the surface. The properties of a fluid are assumed to be constant except for the density in the body force term. In addition, a chemically reactive species is assumed to be emitted from the vertical surface into a hydrodynamic flow field. It diffuses into the fluid, where it undergoes a homogenous chemical reaction. The reaction is assumed to take place entirely in the stream. Then the fully developed flow under the above assumptions through a porous medium is governed by the following set of equations:

$$
\begin{gathered}
\frac{\partial v^{*}}{\partial y^{*}}=0 \\
v^{*} \frac{\partial u^{*}}{\partial y^{*}}=v \frac{\partial^{2} u^{*}}{\partial y^{*}}+g \beta_{T}\left(T^{*}-T_{\infty}^{*}\right)+g \beta_{c}\left(C^{*}-C_{\infty}^{*}\right)-\frac{\sigma B_{0}^{2}}{\rho} u^{*}-\frac{v u^{*}}{k_{p}} \\
v^{*} \frac{\partial T^{*}}{\partial y^{*}}=\frac{k}{\rho C_{p}} \frac{\partial^{2} T^{*}}{\partial y^{* 2}}+\frac{v}{C_{p}}\left(\frac{\partial u^{*}}{\partial y^{*}}\right)^{2}-\frac{v}{\rho C_{p}} \frac{\partial q_{r}}{\partial y^{*}}+\frac{Q_{0}}{\rho C_{p}}\left(T^{*}-T_{\infty}^{*}\right) \\
v^{*} \frac{\partial C^{*}}{\partial y^{*}}=D \frac{\partial^{2} C^{*}}{\partial y^{*^{2}}}-k_{c}\left(C^{*}-C_{\infty}^{*}\right)+D_{1} \frac{\partial^{2} T^{*}}{\partial y^{*^{2}}}
\end{gathered}
$$

The relevant boundary conditions are given as follows

$$
\begin{aligned}
& u^{*}=0, T^{*}=T_{w}, C^{*}=C_{w} \text { at } y=0 \\
& u^{*} \rightarrow 0, T^{*} \rightarrow T_{\infty}, C^{*} \rightarrow C_{\infty} \text { as } y^{*} \rightarrow \infty
\end{aligned}
$$

Eq. (1) gives that

$$
v^{*}=\text { constant }=-v_{0}
$$

In the optically thick limit, the fluid does not absorb its own emitted radiation in which there is no self-absorption, but it does absorb radiation emitted by the boundaries. Cogley et al. [34] have shown that, in the optically thin limit for a non-gray gas near equilibrium, the radiative heat flux is represented by the following form:

$$
\frac{\partial q_{r}}{\partial y^{*}}=4\left(T^{*}-T_{\infty}^{*}\right) \int_{0}^{\infty} K_{\lambda w}\left(\frac{d e_{b \lambda}}{d T^{*}}\right)_{w} d \lambda=4 I_{1}\left(T^{*}-T_{\infty}^{*}\right)
$$

On introducing the following non-dimensional quantities, 


$$
\begin{aligned}
& u=\frac{u^{*}}{v_{0}}, y=\frac{v_{0} y^{*}}{v}, \theta=\frac{T^{*}-T_{\infty}^{*}}{T_{w}-T_{\infty}{ }^{*}}, C=\frac{C^{*}-C_{\infty}^{*}}{C_{w}-C_{\infty}{ }^{*}}, \operatorname{Pr}=\frac{v \rho c_{p}}{k}, S c=\frac{v}{D}, M=\frac{\sigma B_{0}{ }^{2} v}{\rho v_{0}{ }^{2}} \\
& G r=\frac{v g \beta_{T}\left(T_{w}-T_{\infty}^{*}\right)}{v_{0}{ }^{3}}, G m=\frac{v g \beta_{c}\left(C_{w}-C_{\infty}^{*}\right)}{v_{0}{ }^{3}}, E=\frac{v_{0}{ }^{2}}{C_{p}\left(T_{w}-T_{w}{ }^{*}\right)}, k=\frac{v_{0}{ }^{2} k_{p}}{v^{2}}, K r=\frac{v k_{c}}{v_{0}{ }^{2}} \\
& F=\frac{4 I_{1} v}{k v_{0}{ }^{2}}, Q=\frac{Q_{0} v}{\rho c_{p} v_{0}{ }^{2} p r}, S 0=\frac{D_{1}\left(T_{w}-T_{\infty}^{*}\right)}{v\left(C_{w}-C_{\infty}^{*}\right)}
\end{aligned}
$$

the non-dimensional form of the governing Eqs. (2)- (4) reduce to

$$
\begin{aligned}
& u^{\prime \prime}+u^{\prime}-M_{1} u=-G r \theta-G m C \\
& \theta^{\prime \prime}+\operatorname{Pr} \theta^{\prime}=-\operatorname{Pr} E\left(u^{\prime}\right)^{2}+M_{2} \theta \\
& C^{\prime \prime}+S c C^{\prime}=S c K r C-S 0 S c \theta^{\prime \prime}
\end{aligned}
$$

where $M_{1}=M+\frac{1}{k}, M_{2}=F-Q$.

The corresponding boundary conditions are given by

$$
\begin{aligned}
& u=0, \theta=1, C=1 \text { at } y=0 \\
& u \rightarrow 0, \theta \rightarrow 0, C \rightarrow 0 \text { as } y \rightarrow \infty
\end{aligned}
$$

\section{Solution of the problem}

In order to solve the coupled nonlinear system of Eqs. (9)-(11) with the boundary conditions Eq. (12), the following simple perturbation is used. The governing Eqs. (9)-(11) are expanded in powers of Eckert number $\mathrm{E}(<1)$.

$$
\begin{aligned}
& u=u_{0}+E u_{1}+O\left(E^{2}\right) \\
& \theta=\theta_{0}+E \theta_{1}+O\left(E^{2}\right) \\
& C=C_{0}+E C_{1}+O\left(E^{2}\right)
\end{aligned}
$$

Substituting Eq. (13) into Eq. (9)-(11) and equating the coefficient at the terms with the same powers of $E$, and neglecting the terms of the higher order, the following equations are obtained.

Zero order terms:

First order terms:

$$
\begin{gathered}
u_{0}^{\prime \prime}+u_{0}^{\prime}-M_{1} u_{0}=-G r \theta_{0}-G m C_{0} \\
\theta_{0}^{\prime \prime}+\operatorname{Pr} \theta_{0}^{\prime}-M_{2} \theta_{0}=0 \\
C_{0}^{\prime \prime}+S c C_{0}^{\prime}-K r S c C_{0}=-S c S 0 \theta_{0}^{\prime \prime}
\end{gathered}
$$

$$
\begin{gathered}
u_{1}^{\prime \prime}+u_{1}^{\prime}-M_{1} u_{1}=-G r \theta_{1}-G m C_{1} \\
\theta_{1}^{\prime \prime}+\operatorname{Pr} \theta_{1}^{\prime}-M_{2} \theta_{1}=-\operatorname{Pr}\left(u_{0}^{\prime}\right)^{2} \\
C_{1}^{\prime \prime}+S c C_{1}^{\prime}-K r S c C_{1}=-S c S 0 \theta_{1}^{\prime \prime}
\end{gathered}
$$

The corresponding boundary conditions are

$$
\begin{aligned}
& u_{0}=0, u_{1}=0, \theta_{0}=1, \theta_{1}=0, C_{0}=1, C_{1}=0 \text { at } y=0 \\
& u_{0} \rightarrow 0, u_{1} \rightarrow 0, \theta_{0} \rightarrow 0, \theta_{1} \rightarrow 0, C_{0} \rightarrow 0, C_{1} \rightarrow 0 \text { as } y \rightarrow 0
\end{aligned}
$$

Solving Eqs. (14)-(19) under the boundary conditions (20), the following solutions are obtained.

$$
\begin{gathered}
\theta_{0}=e^{-m_{1} y} \\
C_{0}=A_{2} e^{-m_{2} y}-A_{1} e^{-m_{1} y} \\
u_{0}=A_{5} e^{-m_{3} y}-A_{4} e^{-m_{2} y}+A_{3} e^{-m_{1} y} \\
\theta_{1}=A_{12} e^{-m_{4} y}-A_{6} e^{-2 m_{3} y}-A_{7} e^{-2 m_{2} y}-A_{8} e^{-2 m_{1} y}+A_{9} e^{-\left(m_{3}+m_{2}\right) y}+A_{10} e^{-\left(m_{1}+m_{2}\right) y}-A_{11} e^{-\left(m_{1}+m_{3}\right) y}
\end{gathered}
$$




$$
\begin{aligned}
C_{1}= & A_{20} e^{-m_{5} y}-A_{13} e^{-m_{4} y}+A_{14} e^{-2 m_{3} y}+A_{15} e^{-2 m_{2} y}+A_{16} e^{-2 m_{1} y} \\
& -A_{17} e^{-\left(m_{3}+m_{2}\right) y}-A_{18} e^{-\left(m_{1}+m_{2}\right) y}+A_{19} e^{-\left(m_{1}+m_{3}\right) y} \\
u_{1}= & A_{29} e^{-m_{6} y}-A_{21} e^{-m_{5} y}+A_{22} e^{-m_{4} y}+A_{23} e^{-2 m_{3} y}+A_{24} e^{-2 m_{2} y} \\
& +A_{25} e^{-2 m_{1} y}+A_{26} e^{-\left(m_{3}+m_{2}\right) y}+A_{27} e^{-\left(m_{1}+m_{2}\right) y}+A_{28} e^{-\left(m_{1}+m_{3}\right) y}
\end{aligned}
$$

Substituting Eqs. (21)-(26) into Eq. (13), we obtain the velocity, temperature and concentration distribution in the boundary layer as follows:

$$
\begin{aligned}
& U(y, t)=\left[A_{5} e^{-m_{3} y}-A_{4} e^{-m_{2} y}+A_{3} e^{-m_{1} y}\right] \\
& +E\left[\begin{array}{l}
A_{29} e^{-m_{6} y}-A_{21} e^{-m_{5} y}+A_{22} e^{-m_{4} y}+A_{23} e^{-2 m_{3} y}+A_{24} e^{-2 m_{2} y} \\
+A_{25} e^{-2 m_{1} y}+A_{26} e^{-\left(m_{3}+m_{2}\right) y}+A_{27} e^{-\left(m_{1}+m_{2}\right) y}+A_{28} e^{-\left(m_{1}+m_{3}\right) y}
\end{array}\right] \\
& \theta(y, t)=\left(e^{-m_{1} y}\right)+E\left[\begin{array}{l}
A_{12} e^{-m_{4} y}-A_{6} e^{-2 m_{3} y}-A_{7} e^{-2 m_{2} y}-A_{8} e^{-2 m_{1} y} \\
+A_{9} e^{-\left(m_{3}+m_{2}\right) y}+A_{10} e^{-\left(m_{1}+m_{2}\right) y}-A_{11} e^{-\left(m_{1}+m_{3}\right) y}
\end{array}\right] \\
& C(y, t)=\left(A_{2} e^{-m_{2} y}-A_{1} e^{-m_{1} y}\right)+E\left(\begin{array}{l}
A_{20} e^{-m_{5} y}-A_{13} e^{-m_{4} y}+A_{14} e^{-2 m_{3} y}+A_{15} e^{-2 m_{2} y}+A_{16} e^{-2 m_{1} y} \\
-A_{17} e^{-\left(m_{3}+m_{2}\right) y}-A_{18} e^{-\left(m_{1}+m_{2}\right) y}+A_{19} e^{-\left(m_{1}+m_{3}\right) y}
\end{array}\right)
\end{aligned}
$$

The expressions for the constants in the above equations are given in the Appendix.

The skin-friction, Nusselt number and Sherwood number are important physical parameters for this type of boundary layer flow.

Knowing the velocity field, the skin-friction at the plate can be obtained, which in non-dimensional form is given by $\tau=\left(\frac{\partial u}{\partial y}\right)_{y=0}$

$$
\tau=\left(-m_{3} A_{5}+A_{4} m_{2}-m_{1} A_{3}\right)+E\left(\begin{array}{l}
-m_{6} A_{29}+A_{21} m_{5}-m_{4} A_{22}-2 m_{3} A_{23}-2 A_{24} m_{2}-2 m_{1} A_{25} \\
-\left(m_{3}+m_{2}\right) A_{26}-\left(m_{1}+m_{2}\right) A_{27}-\left(m_{3}+m_{1}\right) A_{28}
\end{array}\right)
$$

Knowing the temperature field, the rate of heat transfer coefficient can be obtained, which in the non-dimensional form, in terms of the Nusselt number, is given by $N u=-\left(\frac{\partial \theta}{\partial y}\right)_{y=0}$

$$
N u=-\left(\left(-m_{1}\right)+E\left(\begin{array}{l}
-m_{4} A_{12}+2 A_{6} m_{3}+2 m_{2} A_{7}+2 m_{1} A_{8} \\
-\left(m_{3}+m_{2}\right) A_{9}-\left(m_{1}+m_{2}\right) A_{10}+\left(m_{3}+m_{1}\right) A_{11}
\end{array}\right)\right)
$$

Knowing the concentration field, the rate of mass transfer coefficient can be obtained, which in the non-dimensional form, in terms of the Sherwood number, is given by $S h=-\left(\frac{\partial C}{\partial y}\right)_{y=0}$

$$
S h=-\left(\left(-A_{2} m_{1}+A_{1} m_{1}\right)+E\left(\begin{array}{l}
-m_{5} A_{20}+m_{4} A_{13}-2 A_{14} m_{3}-2 m_{2} A_{15}-2 m_{1} A_{16} \\
+\left(m_{3}+m_{2}\right) A_{17}+\left(m_{1}+m_{2}\right) A_{18}-\left(m_{3}+m_{1}\right) A_{19}
\end{array}\right)\right)
$$

\section{$4 \quad$ Results and Discussion}

In the preceding section, the problem of a steady free-convective flow of a viscous incompressible, thermally radiating and chemically reacting fluid past a semi-infinite plate in the presence of heat generation and Soret was formulated and solved by means of perturbation method. The expressions for the velocity, temperature, and concentration profiles were obtained. To illustrate the behavior of these physical quantities, numerical values of these quantities were computed with respect to the variations in the governing parameters namely, thermal Grashof number $G r$, solutal Grashof number Gm, Prandtl 
number $\operatorname{Pr}$, Schmidt number $S c$, radiation parameter $F$, magnetic parameter $M$, permeability of porous medium $k$, heat generation parameter $Q$, chemical reaction $K r$ and Soret number $S o$.

In the present study the following default parametric values are adopted: $G r=5.0, G m=3.0, M=1.0$, $k=0.5, \operatorname{Pr}=0.71, E=0.01, F=0.5, Q=0.1, S c=0.22, K r=1.0$ and $S o=1.0$. The numerical results are demonstrated through different graphs as well as tables and their results are interpreted physically.

Fig. 1 indicates the fact that an increase in Grashof number $G r$ for heat transfer leads to a rise in the value of velocity due to enhancement in buoyancy force. The plot of velocity profiles for different values of Grashof number for mass transfer $G m$ is given in Fig. 2. It is observed that velocity increases with Grashof number for mass transfer.

For various values of the magnetic parameter $M$, the velocity profiles are plotted in Fig. 3 . It is obvious that the existence of the magnetic field decreases the velocity. Fig. 4 shows the velocity profiles for different values of the permeability of the porous medium parameter $k$. Clearly as $k$ increases the peak values of the velocity tends to increase. Fig. 5(a) displays Prandtl number Pr effect on the velocity field, it is noticed that velocity decreases as Prandtl number $\operatorname{Pr}$ increases. The influence of Prandtl number $\operatorname{Pr}$ on temperature field is shown in Fig. 5(b), it is observed that temperature profiles decrease as Prandtl parameter $\mathrm{Pr}$ increases.

For different values of the radiation parameter $F$ the velocity profiles and temperature profiles are plotted in Figs .6(a) and 6(b). The radiation parameter $F$ defines the relative contribution of conduction heat transfer to thermal radiation transfer. It is obvious that increases in the radiation parameter $F$ result in a decrease in the velocity and temperature within the boundary layer. The effects of heat generation parameter $Q$ on the velocity profiles and temperature profiles are shown in Figs. 7(a) and 7 (b). From these curves, we see that the heat is generated the buoyancy force increases which induces the flow rate to increase giving rise to the increase in the velocity and temperature profiles. .

Fig. 8 depicts the effect of Schmidt number $S c$ on velocity field. This shows that velocity decreases as the Schmidt number $S c$ increases. Physically this is true because along with the increase in Schmidt number $S c$ the viscosity of the fluid increases and hence velocity decreases. A similar effect is noticed in the presence of chemical reaction parameter $K r$ in Fig. 9, and it is quite interesting to observe the opposite reaction near the plate. Fig. 10 shows the concentration profiles across the boundary layer for various values of Schmidt number Sc. It is found that an increase in $S c$ results in a decrease in the concentration distribution. Because the smaller values of $S c$ are equivalent to increasing the chemical molecular diffusivity.

The effect of concentration is presented in Fig. 11, and from this figure it is noticed that concentration decreases with the increase in chemical reaction parameter $K r$. The effect of Soret number So on the concentration profiles is shown in Fig. 12. From this figure, we see that the concentration profile decreases with the increase of $S o$. The effects of various physical parameters on skin-friction coefficient, Nuselt number and Sherwood number are shown in Table. 1 - 3. The behavior of these parameters is self-evident from Table. 1 to 3 and hence they are not discussed any further in order for brevity.

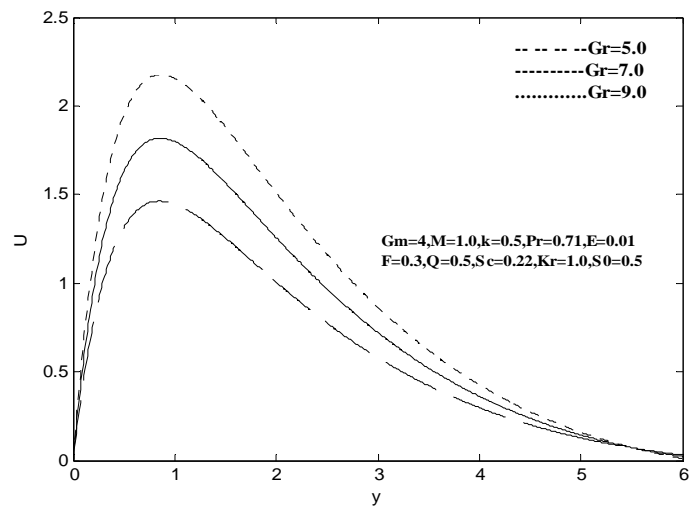

Fig 1. Velocity profiles for various values of $G r$.

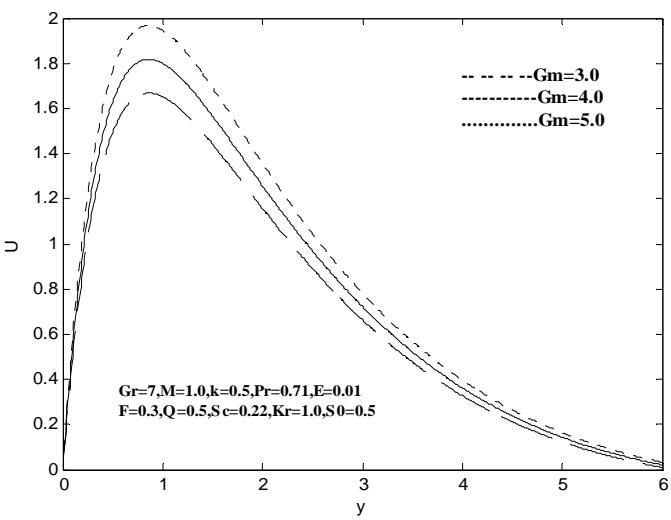

Fig 2.Velocity profiles for various values of $\mathrm{Gm}$. 


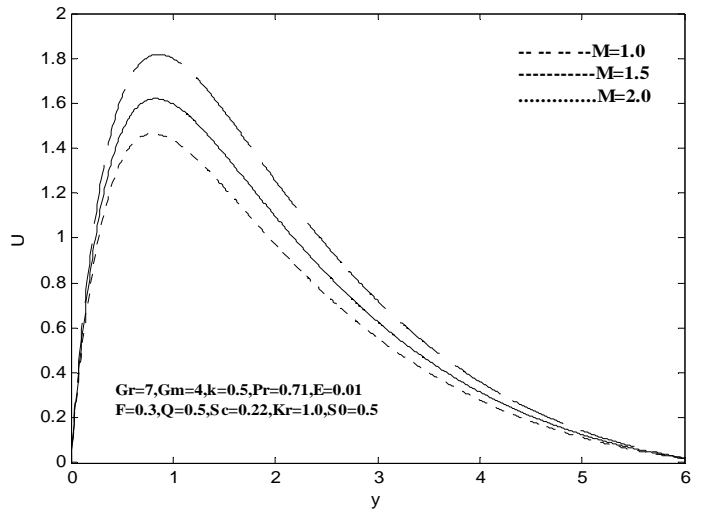

Fig 3. Velocity profiles for various values of $M$.

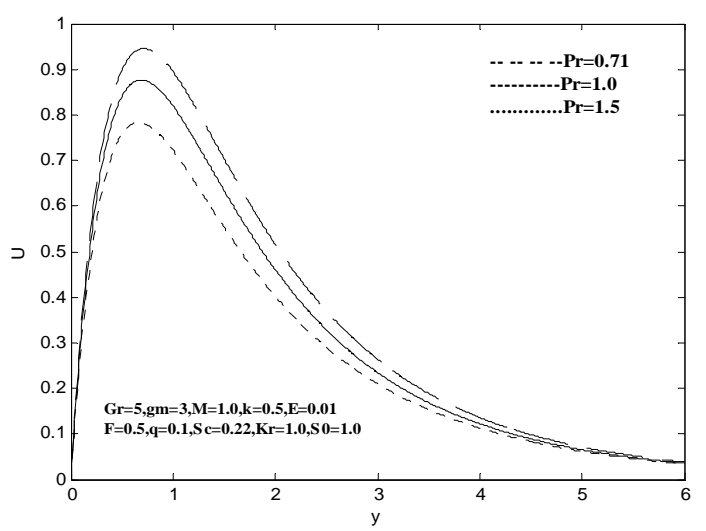

Fig 5(a).Velocity profiles for various values of $\operatorname{Pr}$.

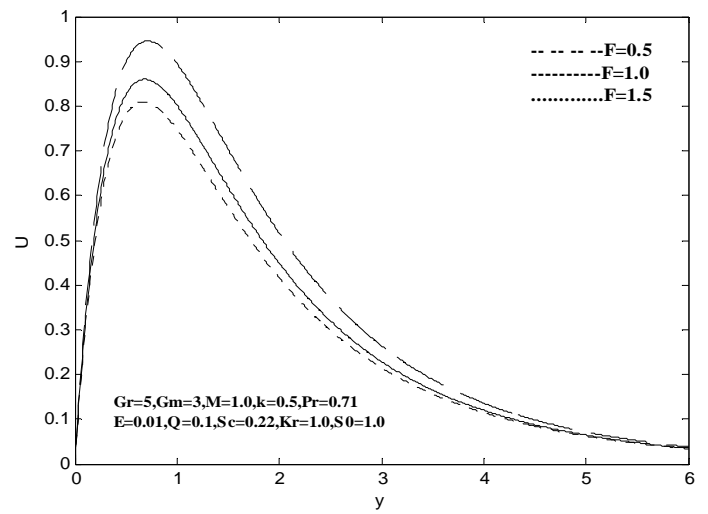

Fig 6(a). Velocity profiles for various values of $F$.

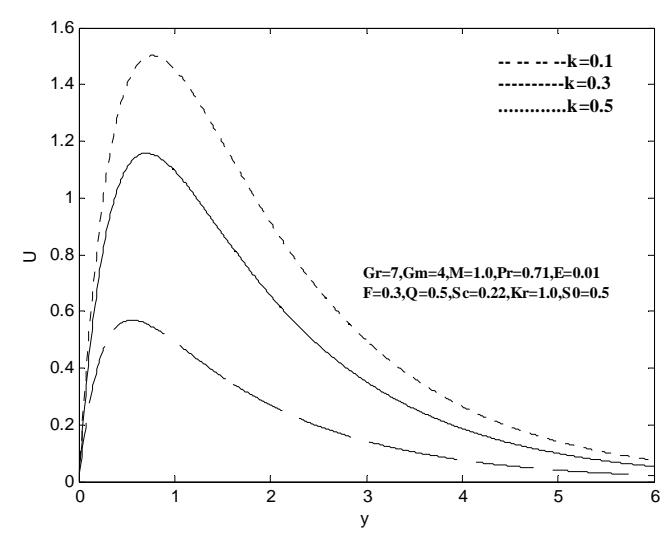

Fig 4. Velocity profiles for various values of $k$.

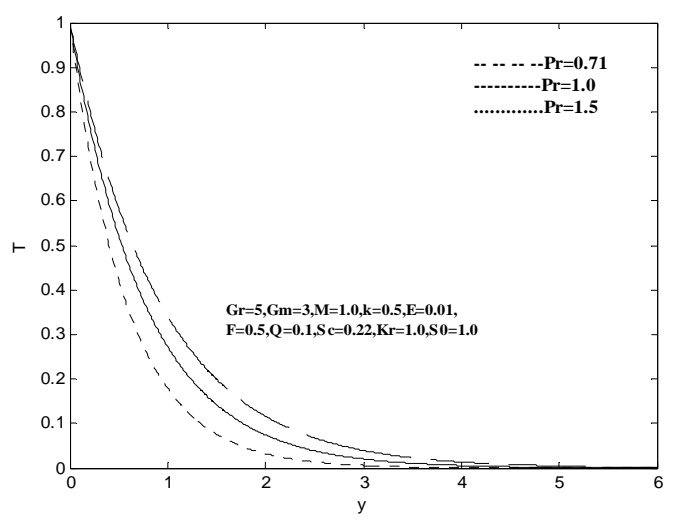

Fig 5(b).Temperature profiles for various values of Pr.

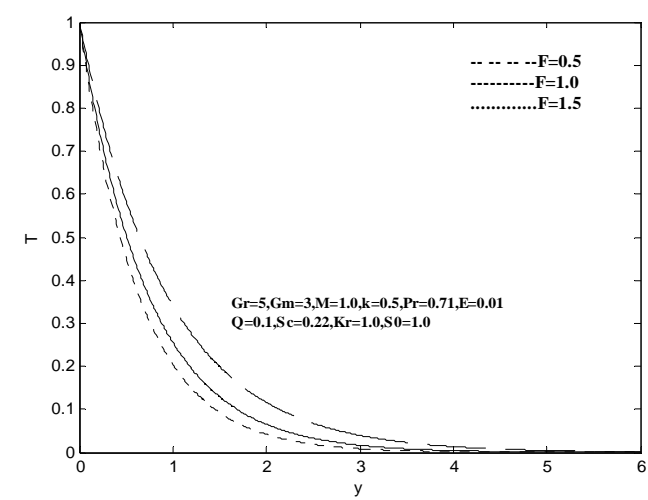

Fig 6(b).Temperature profiles for various values of F. 


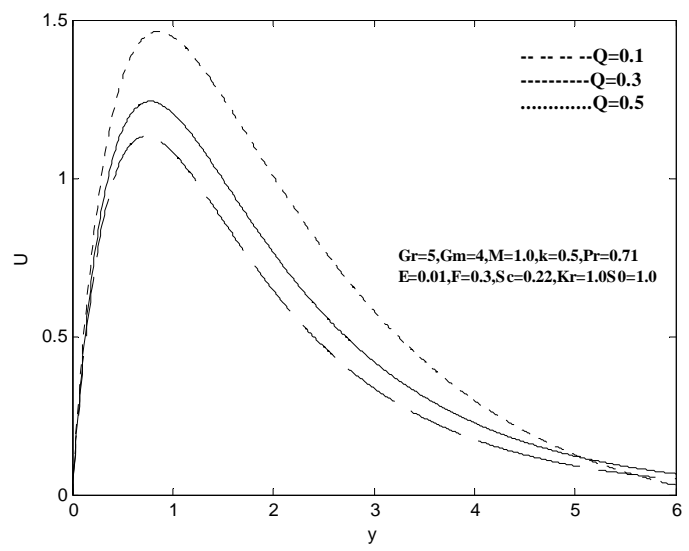

Fig 7(a). Velocity profiles for various values of $Q$.

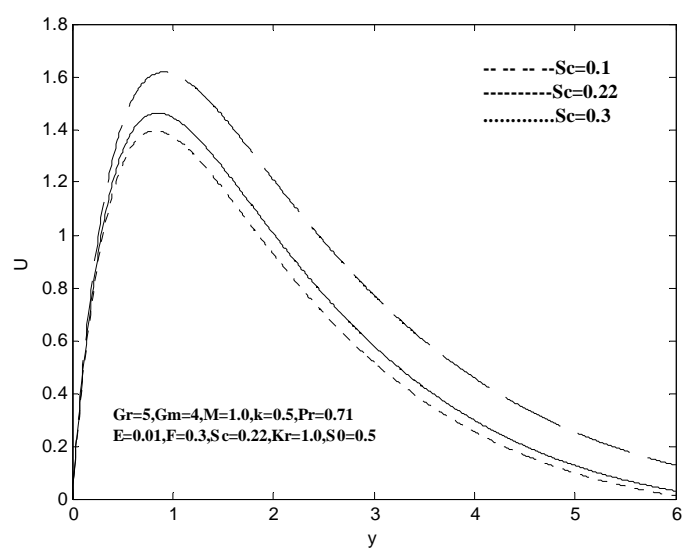

Fig 8. Velocity profiles for various values of $S c$.

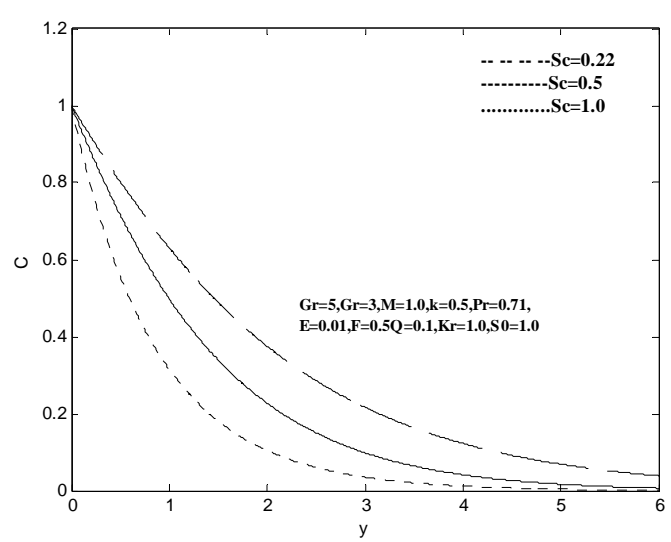

Fig 10. Concentration profiles for various values of Sc.

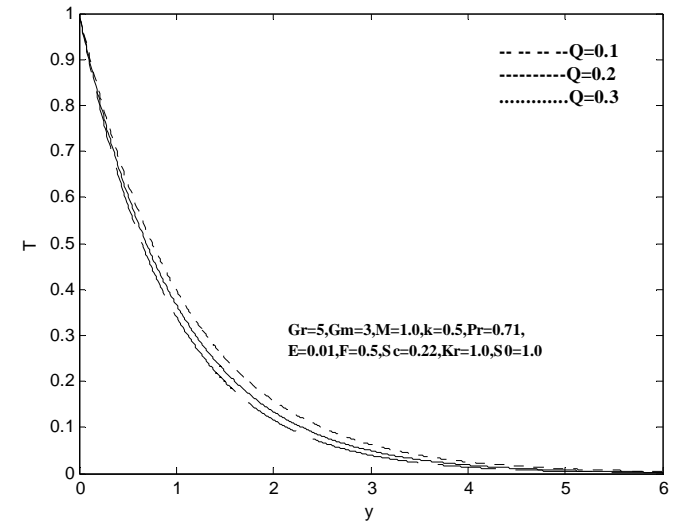

Fig 7(b). Temperature profiles for various values of Q

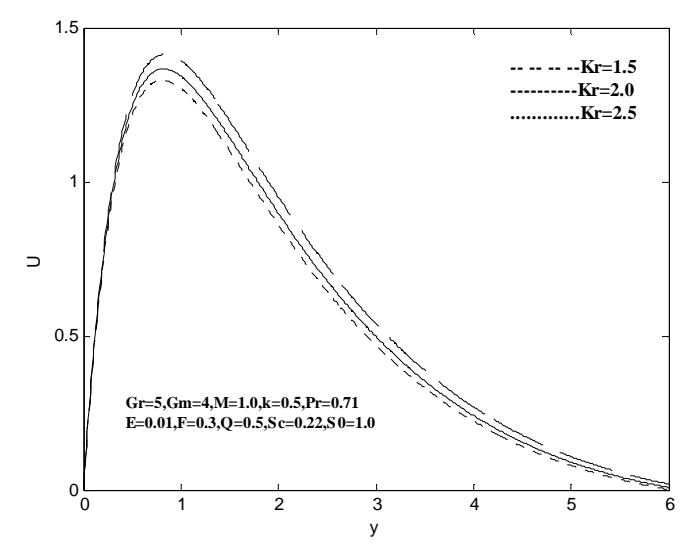

Fig 9. Velocity profiles for various values of $K r$.

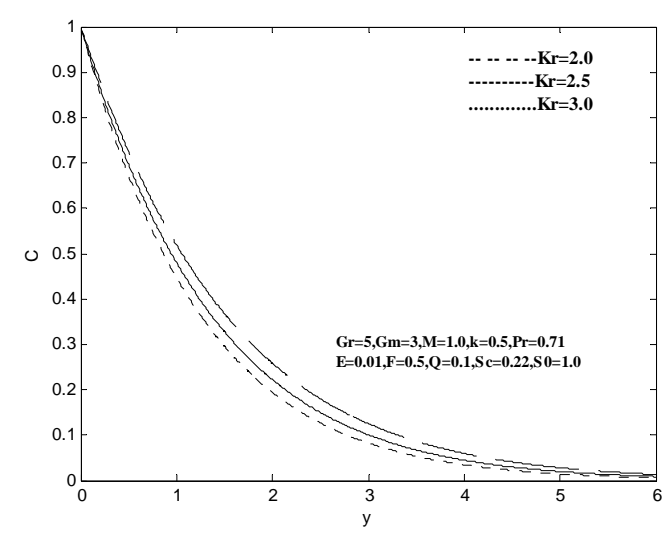

Fig 11. Concentration profiles for various values of Kr 


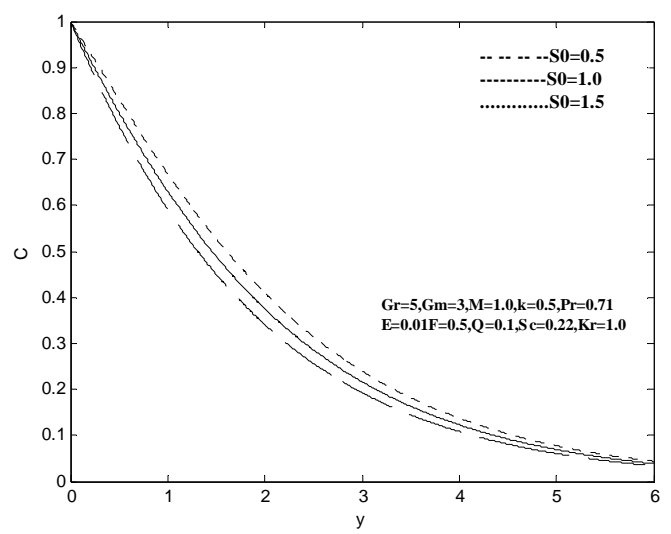

Fig 12. Concentration profiles for various values of $S o$.

Table 1: Effects of various physical parameters on Skin friction for $\operatorname{Pr}=0.71, E=0.01, F=0.5, Q=0.1, S c=0.22$, $K r=1.0, S o=1.0$.

\begin{tabular}{|c|c|c|c|c|c|c|}
\hline$G r$ & $G m$ & $M$ & $K$ & $C f$ & $N u$ & $S h$ \\
\hline 5.0 & 3.0 & 1.0 & 0.5 & 3.8034 & 1.0654 & 0.4182 \\
\hline 7.0 & 3.0 & 1.0 & 0.5 & 4.6475 & 1.0584 & 0.4197 \\
\hline 9.0 & 3.0 & 1.0 & 0.5 & 5.4940 & 1.0501 & 0.4215 \\
\hline 5.0 & 4.0 & 1.0 & 0.5 & 4.3717 & 1.0603 & 0.4193 \\
\hline 5.0 & 5.0 & 1.0 & 0.5 & 4.9400 & 1.0545 & 0.4205 \\
\hline 5.0 & 3.0 & 3.0 & 0.5 & 3.0793 & 1.0717 & 0.4168 \\
\hline 5.0 & 3.0 & 5.0 & 0.5 & 2.6642 & 1.0745 & 0.4162 \\
\hline 5.0 & 3.0 & 1.0 & 0.2 & 2.8483 & 1.0733 & 0.4165 \\
\hline 5.0 & 3.0 & 1.0 & 0.3 & 3.2706 & 1.0703 & 0.4171 \\
\hline
\end{tabular}

Table 2: Effects of various physical parameters on Nusselt number for $G r=5, G m=3, M=1, k=0.5, S c=0.22$, $K r=1, S o=1.0$.

\begin{tabular}{|c|c|c|c|c|c|c|}
\hline$P r$ & $E$ & $F$ & $Q$ & $C f$ & $N u$ & $S h$ \\
\hline 0.71 & 0.01 & 0.5 & 0.1 & 3.8034 & 1.0654 & 0.4182 \\
\hline 1.0 & 0.01 & 0.5 & 0.1 & 3.6406 & 1.2873 & 0.3722 \\
\hline 1.5 & 0.01 & 0.5 & 0.1 & 3.4005 & 1.7071 & 0.2835 \\
\hline 0.71 & 0.02 & 0.5 & 0.1 & 3.8077 & 1.0505 & 0.4214 \\
\hline 0.71 & 0.03 & 0.5 & 0.1 & 3.8120 & 1.0356 & 0.4246 \\
\hline 0.71 & 0.01 & 0.3 & 0.1 & 3.9357 & 0.9091 & 0.4500 \\
\hline 0.71 & 0.01 & 0.4 & 0.1 & 3.8634 & 0.9919 & 0.4332 \\
\hline 0.71 & 0.01 & 0.5 & 0.3 & 3.9357 & 0.9091 & 0.4500 \\
\hline 0.71 & 0.01 & 0.5 & 0.5 & 4.1565 & 0.6891 & 0.4937 \\
\hline
\end{tabular}

Table 3: Effects of various physical parameters on Sherwood number for $G r=5, G m=3, M=1, k=0.5, \operatorname{Pr}=0.71$, $E=0.01, F=0.5, Q=0.1$.

\begin{tabular}{|c|c|c|c|c|c|}
\hline$S c$ & $K r$ & $S o$ & $C f$ & $N u$ & $S h$ \\
\hline 0.22 & 1.0 & 1.0 & 3.8034 & 1.0654 & 0.4182 \\
\hline 0.5 & 1.0 & 1.0 & 3.6062 & 1.0673 & 0.6370 \\
\hline 1.0 & 1.0 & 1.0 & 3.4240 & 1.0689 & 0.9417 \\
\hline
\end{tabular}




\begin{tabular}{|l|l|l|l|l|l|}
\hline 0.66 & 3.0 & 1.0 & 3.5264 & 1.0680 & 0.7889 \\
\hline 0.66 & 5.0 & 1.0 & 3.3820 & 1.0693 & 1.0400 \\
\hline 0.66 & 1.0 & 3.0 & 4.0381 & 1.0628 & 0.0726 \\
\hline 0.66 & 1.0 & 5.0 & 4.2724 & 1.0600 & 0.2704 \\
\hline
\end{tabular}

\section{Conclusions}

This paper studies the effects of the Soret, heat source and chemical reaction on steady MHD free convective boundary layer flow past a porous medium in the presence of thermal radiation and constant suction. The following conclusions can be drawn from the present investigation:

$>$ The velocity profiles increase with the increase of buoyancy forces, permeability parameter and heat source. On the other hand, it decreases with the increase of magnetic parameter and radiation parameter.

$>$ The temperature distributions increase with the increase of heat source.

$>$ The concentration field decreases with the increase of Schmidt number, chemical reaction parameter while it increases with an increase of Soret number.

$>$ It is observed that the increase in the magnetic field parameter is to decrease the skin-friction coefficient, whereas the effect of increasing the value of buoyancy parameter and permeability of porous medium is to increase the value of the skin-friction coefficient.

$>$ Nusselt number decreases with the increase of heat generation parameter and Eckert number.

$>$ Sherwood number increases with the increase of Schmidt number, chemical reaction and Soret number.

\section{Appendix}

$$
\begin{aligned}
& m_{1}=\frac{\operatorname{Pr}+\sqrt{\operatorname{Pr}^{2}+4 M_{2}}}{2}, m_{2}=\frac{S c+\sqrt{S c^{2}+4 K r S c}}{2}, m_{3}=\frac{1+\sqrt{1+4 M_{1}}}{2}, m_{4}=\frac{\operatorname{Pr}+\sqrt{\operatorname{Pr}^{2}+4 M_{2}}}{2}, \\
& m_{5}=\frac{S 0+\sqrt{S c^{2}+4 S c K r}}{2}, m_{6}=\frac{1+\sqrt{1+4 M_{1}}}{2}, \quad A_{1}=\frac{S 0 S c m_{1}^{2}}{m_{1}^{2}-S c m_{1}-K r S c}, A_{2}=1+A_{1} \text {, } \\
& A_{3}=\frac{\left(A_{1} G m-G r\right)}{m_{1}^{2}-m_{1}-M_{1}}, \quad A_{4}=\frac{A_{2} G m}{m_{2}^{2}-m_{2}-M_{1}}, A_{5}=A_{4}-A_{3}, A_{6}=\frac{\operatorname{Pr} m_{3}^{2} A_{5}^{2}}{4 m_{3}^{2}-2 \operatorname{Pr} m_{3}-M_{2}}, \\
& A_{7}=\frac{\operatorname{Pr} m_{2}^{2} A_{4}^{2}}{4 m_{2}{ }^{2}-2 \operatorname{Pr} m_{2}-M_{2}}, \quad A_{8}=\frac{\operatorname{Pr} m_{1}^{2} A_{3}^{2}}{4 m_{1}^{2}-2 \operatorname{Pr} m_{1}-M_{2}}, \quad A_{9}=\frac{2 \operatorname{Pr} m_{3} m_{2} A_{5} A_{4}}{\left(m_{3}+m_{2}\right)^{2}-\operatorname{Pr}\left(m_{3}+m_{2}\right)-M_{2}} \text {, } \\
& A_{10}=\frac{2 \operatorname{Pr} m_{1} m_{2} A_{3} A_{4}}{\left(m_{3}+m_{1}\right)^{2}-\operatorname{Pr}\left(m_{1}+m_{2}\right)-M_{2}}, \quad A_{11}=\frac{2 \operatorname{Pr} m_{3} m_{1} A_{5} A_{3}}{\left(m_{3}+m_{1}\right)^{2}-\operatorname{Pr}\left(m_{3}+m_{1}\right)-M_{2}} \\
& A_{12}=A_{11}-A_{10}-A_{9}+A_{8}+A_{7}+A_{6}, \quad A_{13}=\frac{S 0 S c m_{4}{ }^{2} A_{12}}{m_{4}{ }^{2}-m_{4} S c-K r S c}, \quad A_{14}=\frac{4 S 0 S c m_{3}{ }^{2} A_{6}}{4 m_{3}{ }^{2}-2 m_{3} S c-K r S c}, \\
& A_{15}=\frac{4 S 0 S c m_{2}^{2} A_{7}}{4 m_{2}^{2}-2 m_{2} S c-K r S c}, \quad A_{15}=\frac{4 S 0 S c m_{2}{ }^{2} A_{6}}{4 m_{2}^{2}-2 m_{2} S c-K r S c}, \quad A_{16}=\frac{4 S 0 S c m_{1}^{2} A_{6}}{4 m_{1}^{2}-2 m_{1} S c-K r S c}, \\
& A_{17}=\frac{S 0 S c\left(m_{3}+m_{2}\right)^{2} A_{9}}{\left(m_{3}+m_{2}\right)^{2}-S c\left(m_{3}+m_{2}\right)-K r S c}, \quad A_{18}=\frac{S 0 S c\left(m_{1}+m_{2}\right)^{2} A_{10}}{\left(m_{1}+m_{2}\right)^{2}-S c\left(m_{1}+m_{2}\right)-K r S c},
\end{aligned}
$$




$$
\begin{aligned}
& A_{19}=\frac{S 0 S c\left(m_{3}+m_{1}\right)^{2} A_{11}}{\left(m_{3}+m_{1}\right)^{2}-S c\left(m_{3}+m_{1}\right)-K r S c}, A_{20}=A_{13}-A_{14}-A_{15}-A_{16}+A_{17}+A_{18}-A_{19}, \\
& A_{21}=\frac{A_{20} G m}{m_{5}{ }^{2}-m_{5}-M_{1}}, A_{22}=\frac{\left(A_{13} G m-A_{12} G r\right)}{m_{4}^{2}-m_{4}-M_{1}}, A_{23}=\frac{\left(A_{6} G r-A_{14} G m\right)}{4 m_{3}{ }^{2}-2 m_{3}-M_{1}}, A_{24}=\frac{\left(A_{7} G r-A_{15} G m\right)}{4 m_{2}{ }^{2}-2 m_{2}-M_{1}}, \\
& A_{25}=\frac{\left(A_{8} G r-A_{16} G m\right)}{4 m_{1}^{2}-2 m_{1}-M_{1}}, A_{26}=\frac{\left(A_{17} G m-A_{9} G r\right)}{\left(m_{3}+m_{2}\right)^{2}-\left(m_{3}+m_{2}\right)-M_{1}}, A_{27}=\frac{\left(A_{18} G m-A_{10} G r\right)}{\left(m_{1}+m_{2}\right)^{2}-\left(m_{1}+m_{2}\right)-M_{1}}, \\
& A_{28}=\frac{\left(A_{11} G r-A_{19} G m\right)}{\left(m_{3}+m_{1}\right)^{2}-\left(m_{3}+m_{1}\right)-M_{1}}, A_{29}=A_{21}-A_{22}-A_{23}-A_{24}-A_{25}-A_{26}-A_{27}-A_{28},
\end{aligned}
$$

\section{References}

1. B. C. Sakiadis, "Boundary layer behavior on continuous moving solid surfaces-I. boundary layer equations for two-dimensional and axis-symmetric flow," AIChE J, Vol. 7, pp.26-28, 1961.

2. R.J. Gribben, " proc.Royel.Soclondon," Vol. A287,pp.123-141, 1965.

3. R. J. Goldstein and E. R. G Eckert, "The Steady and Transient Free Convection Boundary Layers on a Uniformly Heated Vertical Plate," Int. J. Heat Mass Transfer, Vol. 1, pp.208-218, 1960.

4. D. M Beard and K. Walters, "Elastico-viscous boundary layer flows two dimensional flow near a stagnation point," Proc.camber.Phil.Soc, Vol. 60, pp.667-674, 1964.

5. V. M. Soundalgekar, S. K Gupta and N. S Birajdra, "Nucl.Eng.Design," Vol.53, pp.309-346, 1979.

6. M. C Raju, S. V. K. Varma and N. A Reddy, "MHD thermal diffison naturel convection between inclined plates in porous medium," Journal of future Engineering and Technology, Vol.6, No.2, pp.45-48, 2012.

7. S. Ahmed, "Induced magnetic field with radiating fluid over a porous vertical plate, analytical study," J Naval Archit Mar Eng, Vol. 7, No. 1, pp. 83-94, 2010.

8. N. K Samira, R. Prasad and M. U. S Reddy, "MHD free convection flow of an elastoviscous fluid past an infinite vertical plate," Astor. Physics and space Science, Vol. 181, pp.125-134, 1990.

9. M. K. Chowdhury and M. N Islam, "MHD free convection flow of visco-elastic fluid past an infinite vertical porous plate," Heat and Mass Transfer, Vol.36, pp.439, 2000.

10. V. Soundalgekhar and H. S Takhar, "Radiative convective flow past a semi-infinite vertical plate," Modeling Measure and control, Vol.51, pp.31-40, 1992.

11. M. C Raju, S. V. K. Varma and N. A Reddy, "Radiation and mass transfer effects on a free convection flow through a porous medium bounded by a vertical surface," Journal of future Engineering and Technology, Vol.7, No.2, pp.7-12, 2012.

12. A. M. Hossain, M. A Alim and D. A. S. Rees, "Effect of Radiation on free convection from a porous a vertical plate," International Journal of Heat and Mass transfer, Vol.42, pp.181-191, 1999.

13. M. A. Abd E- Naby, E. M. E. EI-Barbary and N. Y. Abdelazen, "Finite difference solution of radiation effects on MHD free convection flow on vertical porous plate," Appl.Math.Comput, Vol.151, No.2, pp.327-346, 2004.

14. M. Guria, N. Ghara and R. N. Jana, "Radiation effect on three dimensional vertical channel flow," International Journal of Applied Mechanics and Engineering, Vol. 15, No. 4, pp. 1065-1081, 2010.

15. V. Rajesh and S. V. K. Varma, "Radiation and mass transfer effects on MHD free convection flow past an exponentially accelerated vertical plate with variable temperature," ARPN Journal of Eng. And App. Sci, Vol. 4, No. 6, pp. 20-26, 2009.

16. A. Raptis, "Radiation and flow through a porous medium," Journal of Porous Media, Vol. 4, No. 3, pp. 271-273, 2001.

17. M. C Raju, N. Ananda Reddy and S. V. K. Varma, "Analytical study of MHD free convective dissipative boundary layer flow past a porous vertical surface in the presence of thermal radiation ,chemical reaction and constant suction," Ain Shams Engineering Journal, Vol. 5, pp.1361-1369, 2014. 
18. P. V. Satya Narayana and S. Sravanthi, "Influence of variable permeability on unsteady MHD convection flow past a semi-infinite inclined plate with thermal radiation and chemical reaction," Journal of Energy, Heat and Mass Transfer, Vol.34, pp 143-161, 2012.

19. U. N Das, R. Deka and V. M Soundalgekar, "Effects of mass transfer on flow past an impulsively started infinite vertical plate with constant heat flux and chemical reaction," ForschungimIngenieurwesen, Vol. 60, No. 10, pp. 284-287, 1994.

20. P. V Satya Narayana, "Chemical reaction and thermal radiation effects on an steady MHD free convection flow past an infinite vertical plate with variable suction and heat source or sink," IJMMSA, Vol. 4, pp. 27-45, 2011.

21. A. Mohamed, S. M Abo-Dahab and T. A. Nofal, "Thermal radiation and MHD effects on free convective flow of a polar fluid through a porous medium in the presence of internal heat generation and chemical reaction," Mathematical Problems in Engineering, Vol 2010, Article ID 804719, 27 pages, 2010.

22. F. M. Hady, R. A. Mohamed and A. Mahdy, "MHD free convection flow along a vertical wavy surface with heat generation or absorption effect," International Communications in Heat and Mass Transfer, Vol. 33, No. 10, pp. 1253-1263, 2006.

23. M. S. Alam, M. M Rahman and M. A Sattar, "MHD Free convection heat and mass transfer flow past an inclined surface with heat generation," Thammasat International Journal of Science and Technology, Vol. 11, No. 4, pp. 1-8, 2006.

24. A. J Chamkha, "Heat and mass transfer from MHD flow over a moving permeable cylinder with heat generation or absorption and chemical reaction," Commun. in Numerical Analysis, Article ID cna-00109, 20 pages, 2011.

25. P. Geetha and M. B. K. Moorthy, "Viscous dissipation effect on steady free convection and mass transfer flow past a semi-infinite flat plate," Journal of Computer Science, Vol. 7, No. 7, pp. 1113 - 1118, 2011.

26. S. Suneetha, N. Bhaskar Reddy and V. Ramachandra Prasad, "The thermal radiation effects on MHD free convection flow past an impulsively started vertical plate with variable surface temperature and concentration," Journal of Naval Architecture and Marine engineering, Vol. 2, pp. 57 - 70, 2008.

27. P. V. Satyanarayana, D. Chenna Kesavaiah and S. Venkataramana, "Viscous dissipation and thermal radiation effects on steady MHD convection flow past a semi-infinite vertical permeable moving porous plate," IJMA, 476487, 2011.

28. R. L. Mahajan and B. B. Gebhart, "Viscous dissipation effects in Buoyancy - Induced flows," Int. J. Heat Mass Transfer, Vol. 32, No. 7, pp. 1380 - 1382, 1989.

29. S.Mohammed Ibrahim and K.Suneetha, "Chemical reaction and Soret effects on unsteady MHD flow of a viscoelastic fluid past an impulsively started infinite vertical plate with Heat source," International Journal of Mathematics and Computational Science, Vol.1, No.1, pp.5-14, 2015.

30. M. Bhavana, D. Chenna Kesavaiah and A. Sudhakaraiah, "The Soret effect on free convective unsteady MHD flow over a vertical plate with heat source," International Journal of Innovative Research in Science, Engineering and Technology, Vol.2, No.5, pp.1617-1628, 2013.

31. C. Pavan Kumar, U. Rajeswara Rao and D. R. Prasad Rao, "Chemical reaction and thermo-Diffusion effects on Hydromagentic free convective Walter's Memory flow which constant suction and heat sink," International Journal of Mathematical Archive, Vol.4, No.7, pp. 94-104, 2013.

32. N. Ahmed, "Heat and mass transfer in Hartman flow with Soret effect in presence of a constant heat source," Turkish J of phy, Vol. 33, pp.446-460, 2012.

33. N. Ahmed, "Soret and radiation effects on transient free convection from an impulsively started infinite vertical plate," Journal of Heat transfer, (ASME), Vol. 134, pp.1-9, 2012.

34. A. C. Cogley, W. G. Vincenti and S. E. Gill, "Differential approximation for radiative transfer in a non-gray gas near equilibrium," AIAA J, Vol. 6, pp. 551-553, 1968. 